\title{
Gastric precancerous lesions: heading for an international consensus
}

\author{
R M Genta, M Rugge
}

\section{Summary}

As pathological criteria lie at the foundation for the classification of many diseases, a crucial requisite for such classifications to be valid is that their morphological basis be standardised. Inadequately standardised diagnostic criteria result in unacceptable interobserver variation, a factor that may influence both individual patient care and the evaluation of clinical protocols.

One of the most important goals in gastric diseases today is to establish whether cure of Helicobacter pylori is an effective preventive measure against gastric cancer. To tackle this issue it is necessary to measure reliably intermediate outcomes, specifically gastric atrophy and dysplasia. However, there is little agreement on what gastric atrophy and atrophic gastritis are, and treatment and follow up results obtained at one clinical centre are often radically different from those obtained at another. Similarly, studies that examine the fate of dysplastic lesions in the stomach show a great divergence of outcomes between Europe and North America and Japan, where the concept of dysplasia has different connotations.

To reach a consensus on the definitions and diagnostic criteria for atrophy and atrophic gastritis, a group of gastrointestinal pathologists and gastroenterologists met in Houston, Texas, USA, in February 1998. Substantive progress was made, but several problems remained, and a study aimed at resolving the issues that seem to stand in the way of an international agreement is currently underway. To fulfil the need for a broad discussion on the diagnostic differences of gastric dysplasia and cancer between East and West, an international group of pathologists gathered in Padova, Italy, in the spring of 1998. Their main objectives were: (1) to agree on the definitions of the spectrum of gastric preneoplastic lesions; (2) to establish an international glossary for gastric precancerous lesions; and (3) to test the consensus and eventually generate guidelines useful to clinicians for the development of management strategies. A consensus was achieved on the definition of gastric dysplasia as preinvasive neoplasia. Other validation studies are underway. The experiences achieved in the search for an international consensus on the phenotypes of atrophic gastritis and gastric dysplasia may represent a model in dealing with the new scenario of a modern evidencebased pathology.

\section{Evidence-based gastric pathology}

Evidence-based medicine is a fashionable new term suggesting that the use of diagnostic and therapeutic modalities be based on validated information (the "evidence"). ${ }^{12}$ By equating histological diagnoses with the immutable "evidence" contained in their histological slides, pathologists are inclined to believe they have always practiced evidence-based medicine, long before the term was coined. ${ }^{1}$ Unfortunately, such certitude does not take into account the crucial variable represented by the pathologist himself. As a result, different pathologists may - and often do-interpret the same histological feature inconsistently, or even divergently. For years the accuracy of the morphological interpretation has not be questioned, and pathologists have been considered the repositories of the final truth. As keepers of the evidence they have had a prominent role in defining nosological categories, and most classifications of disease rest on morphological foundations.

In the past two decades or so, however, attention has been drawn to the fact that a histopathological diagnosis is the result of the subjective interpretation of morphological elements. This realisation prompted some wellestablished concepts to be probed. Among the histopathological diagnoses revisited there figured prominently the concept of colonic epithelial dysplasia, ${ }^{3}$ the diagnosis of hepatitis, ${ }^{4}$ and the evaluation of melanocytic and breast neoplasms. ${ }^{56}$ The results of these and other studies have shown that individual judgment plays a major role in the way histopathological evaluations are reached. To minimise the subjective component (interobserver variability) of the histopathological diagnosis, efforts are being made to provide standardised diagnostic criteria to be consistently applied in the diagnostic process (guidelines).

The shortcomings of non-validated pathological classifications may influence the management of individual patients, but they are compounded when flawed criteria presumed to be accurate are applied to the evaluation of clinical protocols. ${ }^{2}$ A pertinent example is provided by studies that address the effect of eradication of $H$ pylori infection on the regression of atrophic gastritis. ${ }^{7-10}$ Because there is little agreement on what gastric atrophy and atrophic gastritis are, results obtained in one clinical centre are unlikely to be reproduced in another. Similarly, studies that examine the fate of dysplastic lesions in the stomach show a great divergence of outcomes between Europe and North America and Japan, where the concept of dysplasia has different connotations. ${ }^{11} 12$

\section{From gastritis to cancer: Correa's cascade}

Based on extensive epidemiological and pathological cohort studies conducted in Colombia, 
as well as on data gathered in Finland, Estonia, and Japan, Pelayo Correa proposed a paradigm of gastric carcinogenesis that has become known as Correa's cascade. ${ }^{13}$ In this model, chronic gastritis progressively evolves to atrophy and intestinal metaplasia. In some subjects the metaplastic epithelium undergoes further genomic and phenotypic disarrangements (dysplasia) and may progress to invasive neoplasia. Recently, the paradigm has been modified to insert $H$ pylori at the beginning of the sequence. ${ }^{14}{ }^{15}$ Although this model is validated and widely accepted, there is still a wide discordance in the histopathological recognition of two of its crucial steps: atrophy and dysplasia. This review outlines some of the international efforts currently underway to establish uniform criteria for the recognition of atrophic gastritis and gastric epithelial dysplasia.

\section{Gastric atrophy is the loss of appropriate} glands

Alterations of the mucosal architecture, loss of glands, fibrosis, and replacement of epithelial elements by an intestinal type metaplastic epithelium ("intestinal metaplasia") occur in the gastric mucosa of a segment of patients infected with $H$ pylori. ${ }^{16}$ The constellation of these features has been variously called "atrophic gastritis" and "gastric atrophy," but these terms remain poorly defined and, consequently, loosely used. In spite of the efforts made in the original Sydney System, ${ }^{17}$ the updated Sydney System, ${ }^{18}$ and related publications to provide definitions, descriptions, and guidelines for the histopathological evaluation of gastric atrophy, several studies have shown that the interobserver variability among pathologists remains unsatisfactory. ${ }^{19-21}$

We believe that much of the disagreement in the recognition of atrophy may be removed by further qualifying the current definition ("loss of glands") and by tackling the problem of the relation between inflammation and glandular loss. For the purpose of this discussion we shall call "appropriate glands" those glands that should normally be present in the mucosa from a given area of the stomach. ${ }^{22}$ In the antrum/ prepyloric mucosa the appropriate (i.e., "native," "normal," or "original") glands are mucous glands; in the corpus the appropriate glands are the oxyntic glands. The cardia can be considered similar to the antrum, whereas a mixture of the two types of glands is found in the transitional zone.

By adding the modifier "appropriate" to the existing "loss of glands," we may then use the term "loss of appropriate glands" to define any situation in which the glands mentioned earlier disappear. The place of the original glands may be occupied by fibrosis or by an "inappropriate" type of glands, namely glands of some other type that should not be in that location. Such "replacement glands" are known as "metaplasia" and may consist of an epithelium with goblet cells, absorptive cells with or without a microvillous brush border, and Paneth cells (intestinal metaplasia); oxyntic glands may be replaced by mucous (pyloric) glands ("pyloric" or "pseudo-pyloric" metaplasia).

\section{Indefinite for atrophy}

Chronic active inflammation of the gastric mucosa is the basic feature of $H$ pylori infection. ${ }^{23} 24$ Dense inflammatory infiltrates (including lymphoid aggregates and follicles) may displace or conceal glands giving the impression of gland loss, or may infiltrate a lamina propria from which glands have indeed vanished. ${ }^{25}$ We propose the term "indefinite for atrophy" to categorise such cases. The introduction of the term "indefinite" is not meant to restrict one's ability to detect or diagnose atrophy. Rather, it is intended to offer a possibility to suspend judgment until the histopathological picture becomes clearer, as happens after cure of $H$ pylori infection.

\section{From lesion to disease}

The term "atrophic gastritis" refers to a group of specific nosological entities that have in common the loss of appropriate glands from one or more compartments of the stomach. Following the re-classification of the updated Sydney System, two main types of atrophic gastritis are now recognised: autoimmune atrophic gastritis (which affects exclusively the gastric corpus), and multifocal atrophic gastritis, an almost universally metaplastic process that involves both the antrum and the corpus. ${ }^{18} 2627$

These conditions were initially described by pathologists who had access to the entire stomach, either resected surgically or obtained from autopsies. ${ }^{28} 29$ To evaluate correctly the topographic distribution and extension of atrophy through the examination of endoscopic biopsy specimens it is imperative that the samples be adequately representative. In this respect, the recommendations of the updated Sydney System (two specimens from the antrum, one from the incisura angularis, and two from the corpus) constitute a minimum requirement. ${ }^{18}{ }^{30}$ The final categorisation of the phenotype of atrophic gastritis should result from a comprehensive evaluation of the lesions detected in each individual biopsy specimen.

When the loss of appropriate glands is detected and properly recognised in a limited portion of the stomach (e.g., a mucosal biopsy specimen, a small stripped segment of mucosa) all we can say is that in that particular sample an atrophy producing process has occurred. There are situations in which even severe histological atrophy in a gastric mucosal sample does not represent atrophic gastritis. The most common instance is when a biopsy specimen is obtained from an intestinalised focus in the antrum of a subject with $H$ pylori infection. A large percentage of such individuals have small foci of intestinal metaplasia in their antral mucosa, whereas the rest of the gastric mucosa has the features of chronic active gastritis without any atrophy or metaplasia. ${ }^{31}$ Another less common situation in which focal atrophy can be found is when a biopsy is obtained from an ulcer scar. Such a biopsy specimen would show the complete 
replacement of glands with fibrous tissue, thus giving the histopathological appearance of severe non-metaplastic atrophy. However, if the cause of the ulcer had been-for example, ingestion of non-steroidal anti-inflammatory drugs, the remainder of the gastric mucosa might be completely normal. These examples illustrate how adequate sampling is critical to the pathologist's ability to discriminate between focal gland loss (the lesion) and the widespread atrophy that characterises atrophic gastritis (the disease).

\section{Atrophy '99}

In an attempt to resolve some of these issues and specifically to reach a consensus on the definitions and diagnostic criteria for atrophy and atrophic gastritis, a group of gastrointestinal pathologists and gastroenterologists met in Houston, Texas, USA, in February 1998. Although substantive progress was made, several problems remained.

One of the main unresolved issued was the relation between atrophy and intestinal metaplasia. A study aimed at resolving the issues that seem to stand in the way of an international agreement is currently underway (Atrophy '99). The participants and their affiliations are listed in the Appendix.

\section{Gastric dysplasia}

Over the past 25 years it has become clear that the malignancy is the final step of progressive phenotypic changes. ${ }^{13} 3233$ Such changes modify the original cellular morphology, eventually generating a biologically new (i.e., $v \varepsilon 0=$ neo-) cell characterised by uncontrolled growth (i.e., $\pi \lambda \alpha \zeta \omega=$ plasia) and the potential to migrate and implant in locations beyond its original fixed site $(\mu \varepsilon \tau \alpha=$ beyond; $1 \sigma \tau \alpha \nu \alpha \iota=$ to stay in place). This biological process has been called multistep or stepwise oncogenesis.

A collection of (mostly unknown) genetic alterations underlies the neoplastic changes of the original cellular phenotype. They vary from cancer to cancer and from subject to subject in quality, number, and timing. The phenotypic counterpart of such genomic alterations consists of a continuum of morphological lesions characterised by progressive dedifferentiation of the histological and cytological structure of the original tissue. In the intestinal district, such a model has been originally studied in the mucosa of the large bowel..$^{34}$ In this context, three main categories of lesions have been distinguished: (a) "true" normal, (b) atypical lesions, mostly polypoid, associated with high risk of cancer evolution, and (c) cancer. ${ }^{3}$ The definition of dysplasia was successfully applied to the intermediate category, also emphasising the increasing cancer risk associated with the increasing degree of atypical phenotypes. ${ }^{36} \mathrm{On}$ the basis of the large bowel model, similar aberrant epithelia have also been described in the stomach mucosa, where they were formerly considered as coexisting with gastric malignancies. ${ }^{36}$

\section{Will the twain ever meet?}

While increasing evidence was becoming available on dysplasia as both a neoplastic and noninvasive alteration, the Eastern and Western schools developed different descriptions and definitions for this category of lesions. In the Eastern scientific community, particularly in Japan, the term "borderline lesions" was applied to identify the morphological alterations covering the boundary between benign and malignancy. Further modification of the terminology (i.e., atypical epithelium, group III lesions) did not result in any significant changes of the initial description of such lesions. This term applied to a spectrum of histological and cytological abnormalities, irrespective of whether or not invasion of the lamina propria had occurred (i.e., irrespective of their restriction within the confines of the basal membrane). In contrast, the Western literature adopted the term "dysplasia" to refer to a neoplastic proliferation confined to the lamina propria of the original glandular structure.

No evidence-based data have been provided on the interobserver consistency among Eastern pathologists in grading and recognising advanced gastric preneoplastic lesions. However, the low level of interobserver agreement reported among Western pathologists in categorising gastric dysplastic lesions would inter alia justify a critical revisiting of the morphological spectrum of the "western gastric dysplasia." As these ideas began to permeate the thinking of Western pathologists, a group of Eastern and Western pathologists evaluated the consistency of the criteria they adopted in categorising gastric pre-malignancies and initial cancer. ${ }^{12}$ This and other studies suggested that these differences could have a major impact not only on the management of individual patients, but also on the international comparisons of cancer incidence, and the validation of cancer preventive strategies. ${ }^{37} 38$

\section{The Padova classification}

To fulfil the increasingly evident need for a broad discussion on the diagnostic differences between East and West, an international group of pathologists (Appendix), gathered in $\mathrm{Pa}-$ dova, Italy, in the spring of 1998.

The main objectives of this conference were: (1) to reach an agreement on the definitions of the spectrum of gastric preneoplastic lesions; (2) to establish a glossary for gastric precancerous lesions that would be consistently useful in clinical practice and scientific research; (3) to evaluate the interobserver agreement on the histological diagnosis of gastric preneoplastic lesions after a consensus was achieved; and (4) to generate guidelines useful to clinicians for the development of management strategies.

A consensus was achieved on the definition of gastric dysplasia as preinvasive neoplasia. Furthermore, the following main categories were agreed upon: 1 , negative for dysplasia; 2 , indefinite for dysplasia; 3, non-invasive neoplasia; 4, suspicious for invasive cancer; 5, gastric cancer. The numerical prefix assigned to each diagnostic category essentially corresponds to 
the diagnostic categories of the Japanese Classification for Gastric Cancer. Within each category, one or more subcategories are hierarchically ordered to cover the spectrum of epithelial alterations. The validation phase of the proposed classification is currently under way.

\section{Consensus conferences in pathology}

In this era of increasingly regulated medical care, diagnostic and therapeutic decisions can no longer rest exclusively on individual physician's experience, but must be guided by evidence-validated knowledge. One of the crucial steps in the development of management protocols (both analytical and interventional) is the standardisation of the histopathological diagnosis. The experiences achieved in the search for an international consensus on the phenotypes of atrophic gastritis and gastric dysplasia may represent a model in dealing with the new scenario of a modern evidencebased pathology.

1 Batstone G. Practising by the evidence: the role of pathology. f Clin Pathol 1997;50:447-8.

Guyatt GH, Sackett DL, Sinclair JC, et al. Users' guides to the medical literature. IX. A method for grading health care recommendations. Evidence-Based Medicine Working Group. $\mathcal{F} A M A$ 1995;274:1800-4

3 Riddell RH, Goldman H, Ransohoff DF, et al. Dysplasia in inflammatory bowel disease: standardized classification with provisional clinical applications. Hum Pathol 1983;14: 931-68.

4 Anonymous. Intraobserver and interobserver variations in liver biopsy interpretation in patients with chronic hepatitis C. The French METAVIR Cooperative Study Group. Hepatology 1994;20:15-20.

5 Farmer ER, Gonin R, Hanna MP. Discordance in the histopathologic diagnosis of melanoma and melanocytic histopathologic diagnosis of melanoma and melanoc

6 Scott MA, Lagios MD, Axelsson K, et al. Ductal carcinoma in situ of the breast: reproducibility of histological subtype in situ of the breast: reproducibility of

7 Borody TJ, Andrews P, Jankiewicz E, et al. Apparent reversal of early gastric mucosal atrophy after triple therapy for Helicobacter pylori. Am f Gastroenterol 1993;88:1266-8.

8 Oberhuber G, Wuendisch T, Rappel S, et al. Significant improvement of atrophy after eradication therapy in atrophic body gastritis. Pathol Res Pract 1998;194:609-13.

9 Van der Hulst RW, van der Ende A, Dekker FW, et al. Effect of Helicobacter pylori eradication on gastritis in relation to cagA: a prospective 1-year follow-up study. Gastroenterology 1997;113:25-30.

10 Farinati F, Foschia F, Di Mario F, et al. H. pylori eradication and gastric precancerous lesions. Gastroenterology 1998; 115:512-14.

11 Konishi F, Muto T, Kamiya J, et al. Histopathologic comparison of colorectal adenomas in English and Japanese patients. Dis Colon Rectum 1984;27:515-18.

12 Schlemper RJ, Itabashi M, Kato Y, et al. Differences in diagnostic criteria for gastric carcinoma between Japanese and nostic criteria for gastric carcinoma between Japa

13 Correa P. A human model of gastric carcinogenesis. Cancer Res 1988;48:3554-60.

14 Correa P. Is gastric carcinoma an infectious disease? $N$ Eng f Med 1991;325:1170-1.

15 Correa P. Human gastric carcinogenesis: a multistep and multifactorial process. First American Cancer Society Award Lecture on Cancer Epidemiology and Prevention. Cancer Res 1992;52:6735-40.

16 Kuipers EJ, Uyterlinde AM, Pena AS, et al. Long-term sequelae of Helicobacter pylori gastritis. Lancet 1995;345: 1525-8.

17 Price AB. The Sydney System: histological division. 7 Gastroenterol Hepatol 1991;6:209-22.
18 Dixon MF, Genta RM, Yardley JH, et al. Classification and grading of gastritis. The updated Sydney System. Intergrading of gastritis. The updationathology of Gastritis, Houston 1994. Am f Surg Pathol 1996;20:1161-81.

19 Andrew A, Wyatt JI, Dixon MF. Observer variation in the assessment of chronic gastritis according to the Sydney system. Histopathology 1994;25:317-22.

20 el-Zimaity HM, Graham DY, al-Assi MT, et al. Interobserver variation in the histopathological assessment of Helicobacter pylori gastritis. Hum Pathol 1996;27:35-41.

21 Genta RM. Gastric atrophy and atrophic gastritis. Nebulous concepts in search of a definition. Aliment Pharmacol Ther 1998;12(suppl 1):S17-23.

22 Owen DA. Normal histology of the stomach. Am f Surg Pathol 1986;10:48-61.

23 Dixon MF. Pathophysiology of Helicobacter pylori infection. Scand f Gastroenterol Suppl 1994;201:7-10.

24 Faigel DO, Furth EE, Childs $M$, et al. Histological predictors of active Helicobacter pylori infection. Dig Dis Sci 1996;41:937-43.

25 Genta RM. Recognizing atrophy: another step toward a classification of gastritis. Am F Surg Pathol 1996;20(suppl 1):S23-30.

26 Correa P. Chronic gastritis: a clinico-pathological classification. Am f Gastroenterol 1988;83:504-9.

27 Rubin CE. Are there three types of Helicobacter pylori gastritis? Gastroenterology. 1997;112:2108-10.

28 Strickland RG, Mackay IR. A reappraisal of the nature and significance of chronic atrophic gastritis. Am 7 Dig Dis 1973;18:426-40.

29 Stemmermann GN. Intestinal metaplasia of the stomach. A status report. Cancer 1994;74:556-64.

30 Rugge M, Cassaro M, Farinati F, et al. Helicobacter pylori and atrophic gastritis: importance of the cagA status. $\mathcal{F}$ Natl Cancer Inst 1996;88:762-3.

31 Genta RM, Robason GO, Graham DY. Inflammatory responses and intensity of Helicobacter pylori infection in patients with duodenal and gastric ulcer: histopathological analysis with a new stain. Acta Histochem Cytochem 1995;28:67-72

32 Farber E. Cancer development and its natural history. A cancer prevention perspective. Cancer 1988;62:1676-9.

33 Farber E. Reversible and irreversible lesions in processes of cancer development. IARC Sci Publ 1980:143-51.

34 Baker SJ, Markowitz S, Fearon ER, et al. Suppression of human colorectal carcinoma cell growth by wild-type p53. Science 1990;249:912-15.

35 Morson BC, Bussey HJ. Magnitude of risk for cancer in patients with colorectal adenomas. Br f Surg 1985; 72(suppl):S23-5.

36 Morson BC, Sobin LH, Grundmann E, et al. Precancerous conditions and epithelial dysplasia in the stomach. $f$ Clin Pathol 1980;33:711-21.

37 Rugge M, Cassaro M, Farinati F, et al. Diagnosis of gastric carcinoma in Japan and western countries. Lancet. 1997;350:448

38 Rugge M, Farinati F, Baffa R, et al. Gastric epithelial dysplasia in the natural history of gastric cancer: a multicenter prospective follow-up study. Interdisciplinary Group on Gastric Epithelial Dysplasia. Gastroenterology 1994;107: $1288-96$

\section{Appendix}

The Atrophy '99 Working Group: Pelayo Correa, New Orleans, LA, USA; Michael F Dixon, Leeds, UK; Roberto Fiocca, Genova, Italy; Jean-François Fléjou, Paris, France; Robert M Genta, Houston, Texas, USA; Rodger C Haggitt, Seattle, WA, USA; Takanori Hattori, Shiga, Japan; Juan Lechago, Houston, TX, USA; Massimo Rugge, Padova, Italy; Pentti Sipponen, Helsinki, Finland; Enrico Solcia, Pavia, Italy; Hidenobu Watanabe, Niigata, Japan.

The Padova Panel: Pelayo Correa, New Orleans, LA, USA; Michael F Dixon, Leeds, UK; Takanori Hattori, Shiga, Japan; Klaus J Lewin, Los Angeles, CA, USA; Robert H Riddell, Hamilton, Ontario, Canada; Massimo Rugge, Padova, Italy; Pentti Sipponen, Helsinki, Finland; Hidenobu Watanabe, Niigata, Japan. 